\title{
Notes on the occurrence of Ipomoea acanthocarpa and Ipomoea laxiflora (Convolvulaceae) in India
}

\author{
Kattee A.V. ${ }^{1}$, Patil C.R. ${ }^{2}$, Patel S.L. ${ }^{3}$, Kahalkar V.I. ${ }^{4}$ \& V.B. Shimpale ${ }^{1 *}$ \\ ${ }^{1}$ Department of Botany, The New College, Kolhapur district, Maharashtra - 416 012, India. \\ 2Department of Botany, D.K.A.S.C. College, Ichalkaranji, Kolhapur district, Maharashtra - 416 115, India. \\ ${ }^{3}$ Department of Botany, Government College of Daman (UT), Nani Daman, Gujarat - 396 210, India. \\ ${ }^{4}$ Mahatma Gandhi Arts Science and Late N. P. Commerce College, Armori, Gadchiroli district, Maharashtra - 441 208, India. \\ *E-mail: shimpale@yahoo.com
}

\begin{abstract}
Ipomoea acanthocarpa (Choisy) Hochst. ex Schweinf. \& Asch. an African species is reported for the first time from India. Ipomoea laxiflora H.J.Chowdhery \& Debta hitherto recorded from North India, is reported for the first time from Peninsular India. Detailed description, illustrations, photographs and taxonomic notes for both the species are provided.
\end{abstract}

Keywords: Convolvulaceae, India, Ipomoea acanthocarpa, I. laxiflora, New record.

\section{Introduction}

Ipomoea L., with about 650 species, is the largest genus of the family Convolvulaceae (Mabberley, 2017). It is distributed in the tropical and subtropical regions of the world. In India the genus is known to have $c .63$ species (Shimpale et al., 2014). As a part of the ongoing taxonomic revision on the genus Ipomoea in India, authors collected two interesting species, one from Gujarat state and the other from Maharashtra state. After critical examinations and scrutiny with pertinent literature (Hooker, 1883; Cooke, 1905; Hutchinson \& Dalziel, 1972; Shah, 1978; Johri, 1983; Fang \& Staples, 1995; Biju, 1997; Wood et al., 2015) and herbarium consultation, it was concluded that the species collected from Valsad district of Gujarat state is I. acanthocarpa (Choisy) Hochst. ex Schweinf. \& Asch., a species so far reported from Tropical Africa. Hence, the present collection forms a new distributional record for India. While the species collected from Maharashtra state is I. laxiflora H.J.Chowdhery \& Debta, a recently described Himalayan species endemic to North India (Chowdhery \& Debta, 2009; Singh et al., 2015). The present collection outside its type locality indicate an extended

Received: 28.8.2018; Revised \& Accepted: 11.05.2019 Published Online: 30.09.2019 distriution in Peninsular India. A detailed description for both the species along with colour photoplates, illustrations and relevant notes are provided to facilitate easy identification.

Ipomoea acanthocarpa (Choisy) Hochst. ex Schweinf. \& Asch., Beitr. Fl. Aethiop. 277. 1867. Calonyction acanthocarpum Choisy, Prodr. 9: 346. 1845. Type: SUDAN, s.l., s.d., C.G.T. Kotschy 269, (holo P00434126 digital image!; iso K000097123 digital image!).

Figs. 1 \& 2

Perennial climbers with milky latex, c. $5 \mathrm{~m}$ long. Stems twinning, angular, rooting at nodes, tuberculate, brownish when mature; branches hairy at the base. Leaves 5-10 × 6-12 cm, entire, glabrous, cordate at base, acuminate at apex; margins entire or undulate; veins distinct on both surfaces. Petioles 6-12 cm long, tubercled at base, glabrous. Cymes monochasial, axillary, usually 1-5-flowered; peduncles 2-5 cm long, swollen at tip, hairy at base. Pedicels $0.6-1 \mathrm{~cm}$ long, elongated in fruit, slightly tubercled, glabrous; bracts small, caducous, c. $2 \times 1 \mathrm{~mm}$, with distinct veins, glabrous. Sepals 5, sub-equal, 0.6-1 × 0.4$0.8 \mathrm{~cm}$, faintly veined; outer two sepals verrucate, brownish green, smaller; inner three sepals slightly larger than outer, smooth, papery along margins, glabrous. Corolla 5-lobed, 2-3 cm long, funnelshaped; tube c. 1.5-2 cm long, dark purple inside; lobes $c .2 \mathrm{~cm}$ long, apiculate, glabrous, mid-petaline bands pink-purple, shallowly apiculate. Stamens 5; filaments sub-equal, 5-8 mm long, included, attached at c. $2 \mathrm{~mm}$ above the base of tube, hairy at base; anthers c. $0.3 \mathrm{~cm}$ long, basifixed. Ovary ovoid, c. $3 \times 1 \mathrm{~mm}$, glabrous, 4-locular, one ovule in each locule; style c. $6 \mathrm{~mm}$ long, slender, gradually narrowed towards the apex, glabrous; stigma $c .0 .2$ $\times 0.1 \mathrm{~cm}$, bilobed. Capsules sub-globose to ovoid, 


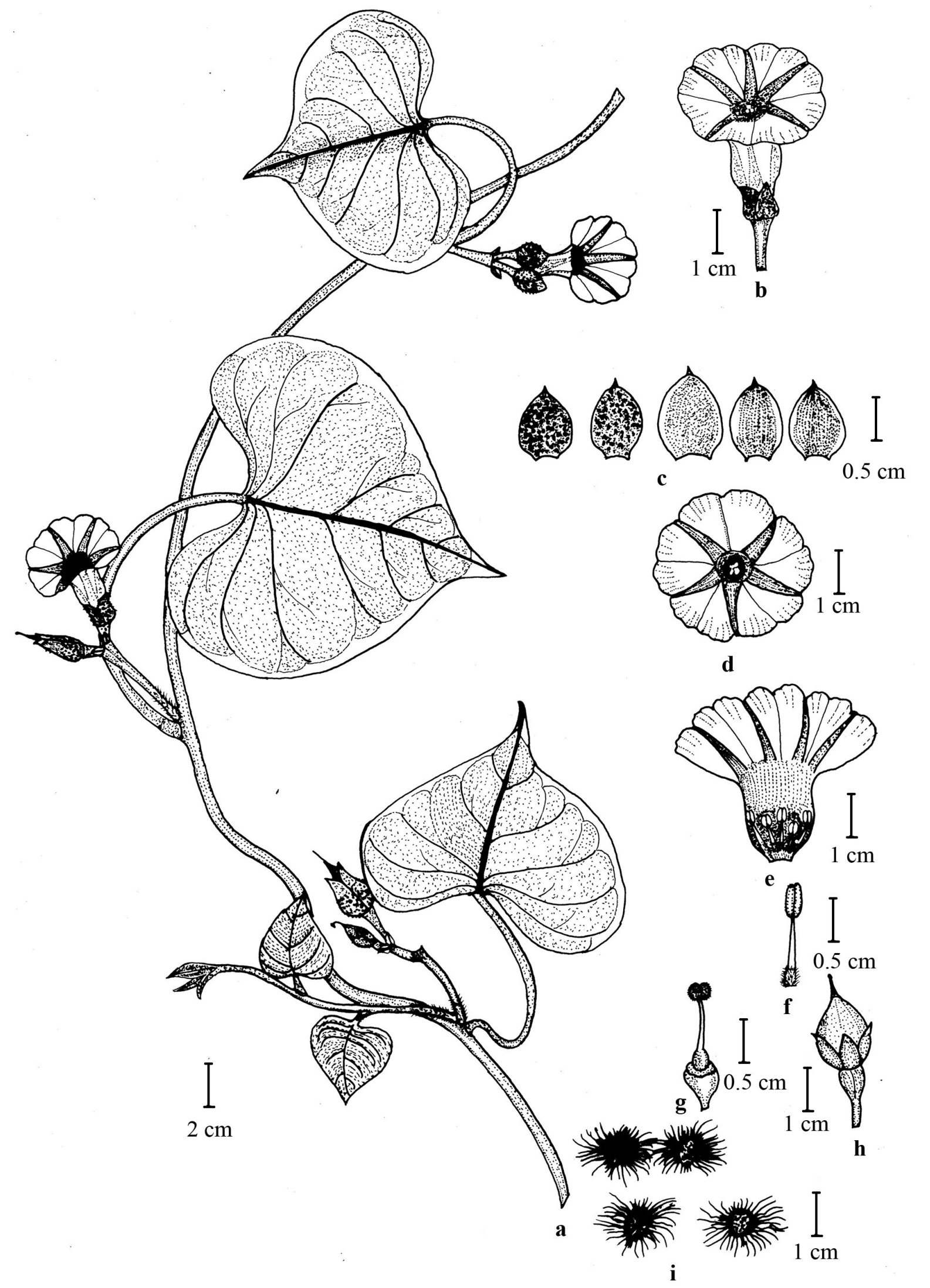

Fig. 1. Ipomoea acanthocarpa (Choisy) Hochst. ex Schweinf. \& Asch.: a. Flowering twig; b. Flower; c. Sepals; d. Corolla front view; e. Corolla split open; f. Stamen; g. Gynoecium; h. Capsule; i. Seeds. 
9-10 mm long, glabrous, brownish, 4-valved, mucronate with persistent stylar beak; stylar beak $c$. $7 \mathrm{~mm}$ long, with spinous apex; seeds $4, c .5 .5 \mathrm{~mm}$ long, grey, pilose at margins.

Flowering \& fruiting: October-March.

Habitat: Along roadsides usually in muddy soil along with I. triloba L. and I. muricata (L.)Jacq. During the present investigation 60-70 individuals were observed in a single population.

Distribution: Tropical Africa, now in India.

Specimen examined: INDIA, Gujarat, Valsad district, Makadban Village, 04.10.2017, Kattee \& Shimpale 1404, 1405 (The New College Herbarium, SUK!).

Ipomoea laxiflora H.J.Chowdhery \& Debta, Indian J. Forest. 32(1): 120.2009. Singh et al., Indian J. Forestry 34(3): 335-338. 2011. Type: INDIA,
Uttarakhand, Dehra Dun district, Kaulagarh Road, Botanical Survey of India Campus, $750 \mathrm{~m}$, 28.09.2008. H.J. Chowdhery 108601 (holo BSD!; iso CAL0000018586!).

Figs. 3 \& 4

Annual climbers, up to $5 \mathrm{~m}$. long; stems purplish green, quadrangular, sparsely hairy at nodes. Leaves variable, simple, entire, 5-12 × 5-10 cm, glabrous, distinctly trilobed; lobes shallow or sharp; petioles c. $12 \mathrm{~cm}$ long, glabrous. Cymes lax, 3-7-flowered; peduncles 6-8 cm long, slightly verrucose, swollen at apex; pedicels $2.5-3 \mathrm{~mm}$ long, quadrangular, glabrous, elongated in fruits. Bracts linear, caducous, c. 3-4 mm long. Calyx 5, sub-equal, $0.8-1 \times 0.2-0.3 \mathrm{~cm}$, faintly veined, purplish at apex, glabrous. Corolla funnel-shaped, c. $1.5 \times 1.2 \mathrm{~cm}$; limb 5-lobed; lobes apiculate. Stamens 5; filaments unequal, included, $0.7-0.8 \mathrm{~cm}$ long, hairy at base. Ovary c. $1 \times 1.5 \mathrm{~mm}$, glabrous;
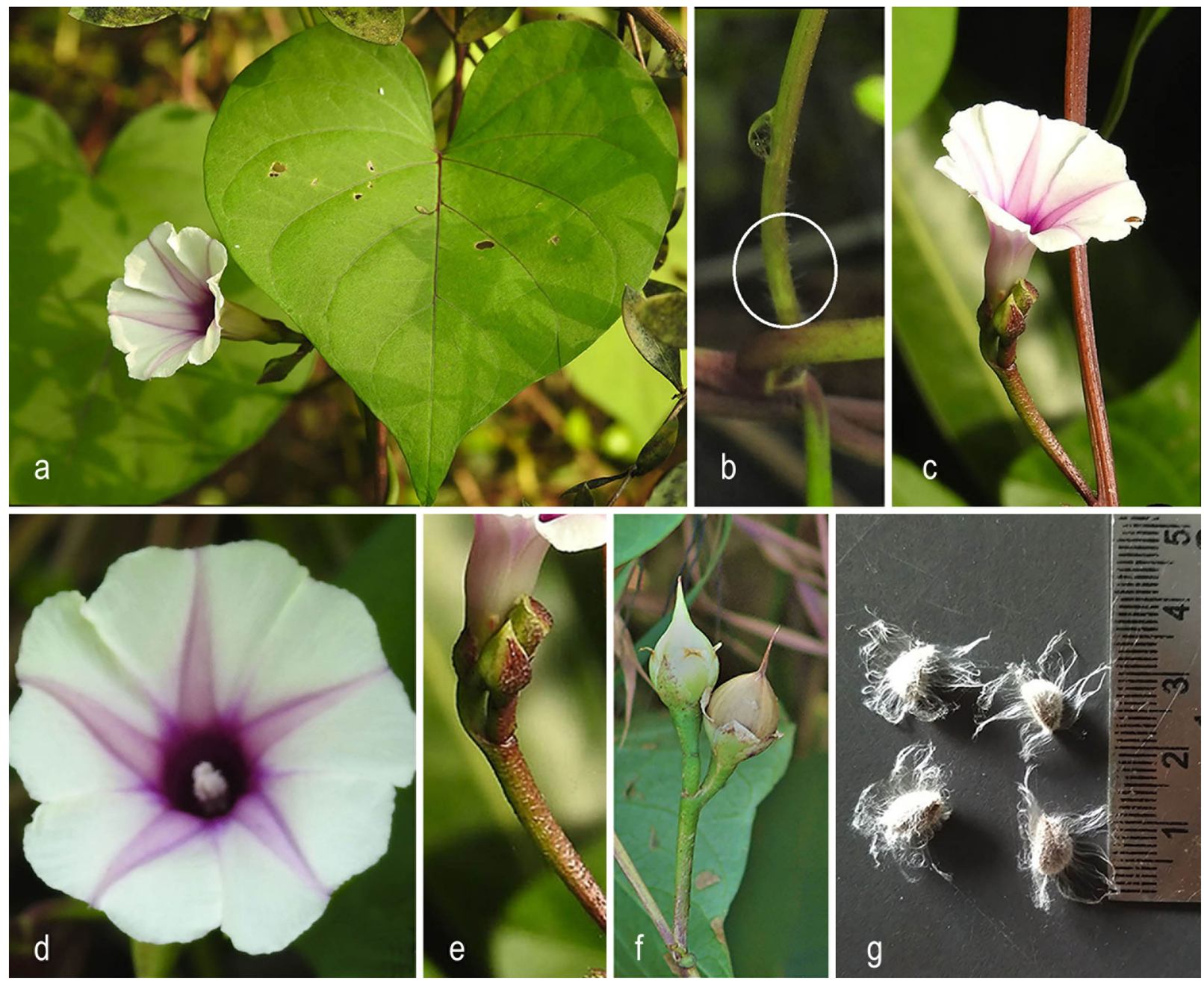

Fig. 2. Ipomoea acanthocarpa (Choisy) Hochst. ex Schweinf. \& Asch.: a. Flowering twig; b. Axillary branch with hairy base; c. Flower; d. Corolla front view; e. Sepals; f. Capsules; g. Seeds. 


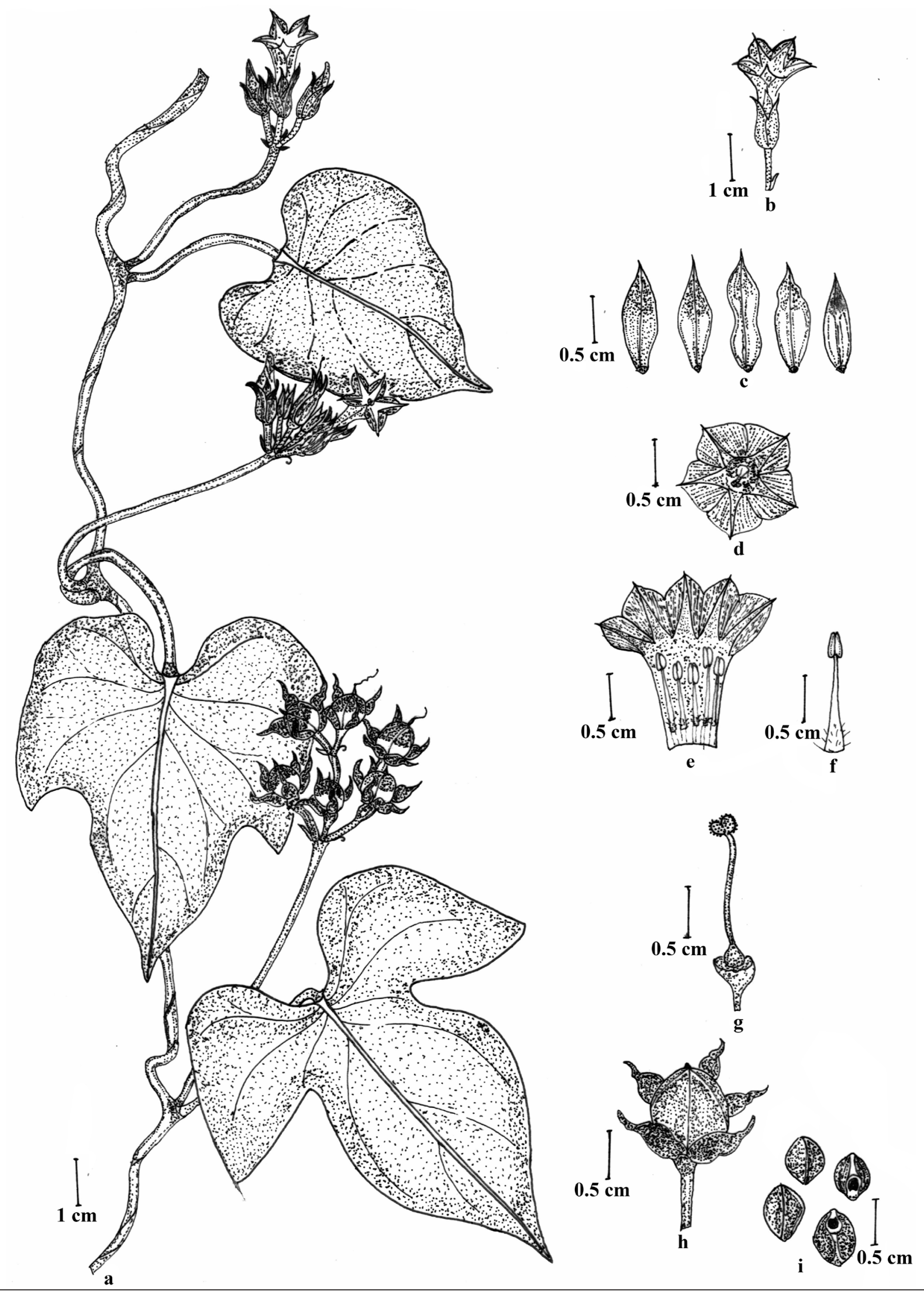

Fig. 3. Ipomoea laxiflora H.J.Chowdhery \& Debta: a. Flowering twig; b. Flower; c. Sepals; d. Corolla front view; e. Corolla split open; f. Stamen; g. Gynoecium; h. Capsule; i. Seeds. 
style c. $1 \mathrm{~cm}$ long; stigma unlobed or 2-lobed. Capsules ovoid, c. $5 \times 6 \mathrm{~mm}$, 4-valved, with purple tinge when young, glabrous; seeds $c .4 \times 4 \mathrm{~mm}$, brownish-black, glabrous.

Flowering \& fruiting: September-October.

Distribution: India.
Specimens examined: INDIA, Maharashtra, Kolhapur district, Ichalkaranji, 02.10.2016, Kattee E Shimpale 1541, 1542, 1543; Gadchiroli district, Gadchiroli, 15.10.2016, Kattee \& Shimpale 1544, 1545 (The New College Herbarium!, SUK!).
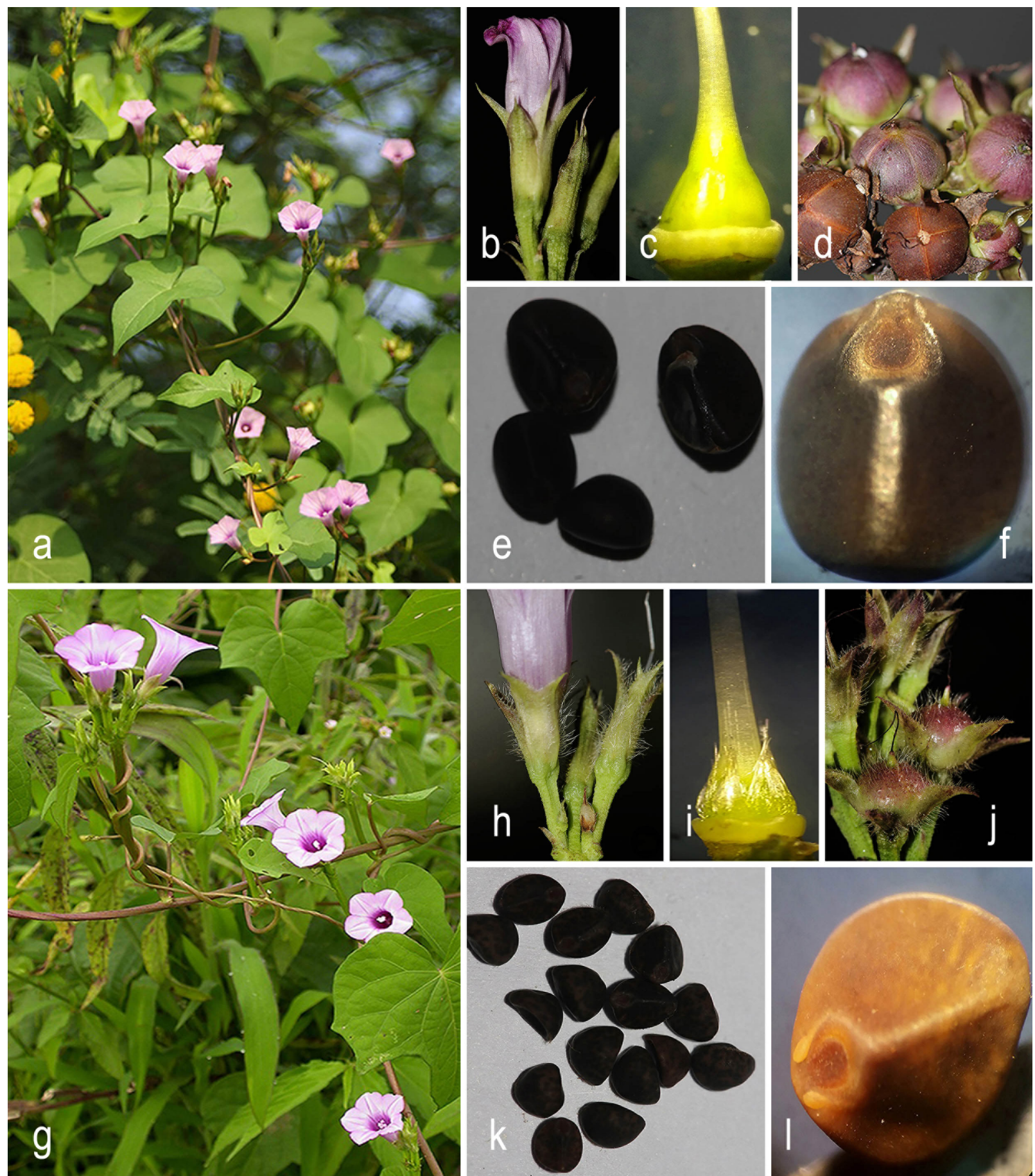

Fig. 4. Ipomoea laxiflora H.J.Chowdhery \& Debta (a-f): a. Habit; b. Sepals; c. Ovary; d. Capsules; e. Seeds; f. Seed -enlarged view. Ipomoea triloba L. (g-l): g. Habit; h. Sepals; i. Ovary; j. Capsules; k. Seeds; I. Seed-enlarged view. 
Table 1. Comparison of distinguishing characters of I. laxiflora and I. triloba

\begin{tabular}{|l|l|l|}
\hline Character & I. triloba L. & I. laxiflora H.J.Chowdhery \& Debta \\
\hline Cymes & Densely flowered & Laxly flowered \\
\hline Ovary & Densely pubescent & Glabrous \\
\hline Stigma & 2-lobed & Unlobed or slightly 2-lobed \\
\hline Fruit & Bristly hairy & Glabrous \\
\hline
\end{tabular}

Notes: This species is close to I. triloba, a wide spread species, the differences between them are given in Table 1. The specimens collected from Maharashtra state show variation in leaf size, inflorescence (from solitary to 5-7-flowered) and stigma slightly bilobed.

\section{Acknowledgements}

Authors are thankful to the Principal, The New College, Kolhapur; Government College of Daman (UT) Nani Daman, Gujarat; Mahatma Gandhi Arts, Science and Late N. P. Commerce College, Armori, Gadchiroli for facilities. AVK is thankful to BARTI, Pune for financial support.

\section{Literature Cited}

BIJU S.D. 1997. Taxonomic and morphologic studies in family Convolvulaceae of southern Peninsular India. Ph. D. Thesis, University of Calicut (Unpublished).

CHOWDHERY H.J. \& M.R. DEBTA 2009. A new species of Ipomoea L. (Convolvulaceae) from India. Indian Journal of Forestry 32(1): 119-121.

COOKE T. 1905. The Flora of the Presidency of Bombay. Volume 2. Taylor \& Francis, London. pp. 222-261.

FANG R.C. \& G. STAPLES 1995. Convolvulaceae In: WU Z., RAVEN P.H. \& D.Y. HONG (Eds.), Flora of China. Volume 16. Missouri Botanical Garden Press \& Science Press (Beijing). pp. 271-328.
HOOKER J.D. 1883. The Flora of British India. Volume 4. L. Reeve \& Co. Ltd., London. pp. 196-216.

HUTCHINSON J. \& M.J. DALZIEL 1972. Flora of West Tropical Africa. Crown Agencies for overseas Government and Administrations, London.

JOHRI S.C. 1983. Genus Ipomoea L. in India. Ph. D. Thesis, University of Rajasthan (Unpublished).

MABBERLEY D.J. 2017. The Plant-Book: A portable dictionary of plants, their classification and uses. Fourth edition. Cambridge University Press, Cambridge.

SHIMPALE V.B., KARE M.A., LONDHE D.K. \& A.S. BHUKTAR. 2014. On the occurrence of Ipomoea tenuipes (Convolvulaceae) in India. Rheedea 24(2): 117119.

SINGH A.K., SAHU R.K. \& M. SRIVASTAVA 2011. On the occurrence of Ipomoea laxiflora in Uttar Pradesh. Indian Journal of Forestry 34(3): 335-338.

SINGH P., KARTHIKEYAN K., LAKSHMI NARASIMHAN P. \& S.S. DASH 2015. Endemic Vascular Plants of India. Botanical Survey of India, Kolkata, p. 144.

SHAH G.L. 1978. Flora of Gujarat State Volume 1. Sardar Patel University, Anand.

WOOD R.I.J., CARINE M.A., HARRIS D., WILKIN P., WILLIAMS B. \& R.W. SCOTLAND 2015. Ipomoea (Convolvulaceae) in Bolivia. Kew Bulletin 70: 31. 